\title{
Immunodetection of FGFI-2 and FGFR I-2 indicates that these proteins disappear in the wound epidermis and blastema of the scarring limb in lizard
}

\begin{abstract}
In lizards while the tail broadly regenerates after amputation, the limb generally forms short scarring outgrowths. FGFs are important growth factors that are present in regenerating tissues and appear to stimulate tail regeneration in lizards. In the limb, immunohistochemistry at 15-16days post-amputation indicates that FGFs and FGFR1-2 are present with different intensity in the wound (regenerating) epidermis and sparsely in connective, muscle and cartilaginous cells of the healing tissues of the stump. However the immunofluorescence study indicate that FGF and FGF1-2 receptors are little or no immunodetectable at 25-26days post-amputation, especially in the wound epidermis, when the limb blastema is turning into a scarring connective tissue of the short limb outgrowth. Western blot analysis shows no low MW bands for FGF1-2 and altered or degraded bands of FGFR1-2 in the limb at 25-26days postamputation when scarring is taking place. The present observations indicate that the disappearance of these proteins is related to loss of cell proliferation that leads to the failure of limb regeneration in lizards. The study further stress the essential role of FGFs and their receptors in the stimulation of organ regeneration in lizards and likely in other amniotes.
\end{abstract}

Keywords: lizard, limb, regeneration, scarring, FGFs, FGFR1. immunohistochemistry
Volume I Issue I - 2017

Lorenzo Alibardi

Department of Biology, University of Bologna, Italy

Correspondence: Lorenzo Alibardi, Department of Biology, University of Bologna, via Selmi 3, 40I26, Bologna, Italy, Email lorenzo.alibardi@unibo.it

Received: April 26, 2017 | Published: May 03, 2017

\section{Introduction}

Among amniotes only lizards are capable of regenerating the tail after amputation but not the limb or digits that instead forms a scar or tail-like outgrowths. ${ }^{1-8}$ The initial phase of limb healing determines the re-epithelialization of the stump and the accumulation of a bulk of connective cells forming a small soft mound termed limb blastema. The microscopic studies of the blastema have shown the presence of few mesenchymal cells accumulating over the injured tissues and instead a massive infiltration of granulocytes and macrophages during the first 3 weeks post-amputation. These cells determine an intense inflammatory reaction that is retained as the main cause of the lack of limb regeneration, as it has been recently indicated also by a transcriptome analysis. ${ }^{9}$ One of the consequences of the intense inflammation is the stimulation of the differentiation of a dense, irregular connective tissue while the amputated bone a cartilaginous cup seals the damage in the bone tissue. The final result is therefore the rapid transition from a soft blastema to a denser, harder, scaled, and flat or short scarring outgrowths. ${ }^{3,6-8}$

In particular it is believed that inflammation impedes the formation/ maintenance of an Apical Epidermal Peg (AEP) at the tip of the wound epidermis of the limb, while keratinocytes rapidly differentiate into a mature epidermis. ${ }^{6-9}$ The AEP is retained essential to maintain tail regeneration, and is linked to the production of Wintless protein (Wnt) and Fibroblast Growth Factors (FGFs). Previous studies have indicated that FGF1-2 tend to disappear in the epidermis of the scarring limb blastema, ${ }^{10}$ suggesting that inflammation depresses the FGF signaling pathway and the maintenance of an AEP that is needed for maintaining tail regeneration. The use of FGFR1 antagonists also reduces or eliminates regeneration in lizards if administered before or at the blastema stage. ${ }^{11-13}$ Finally, recent transcriptome studies have also shown the up-regulation of FGFs and their receptors in the regenerating tail ${ }^{14}$ while these are absent in the scarring limb. ${ }^{9}$ The present study extends these initial findings on FGF1-2 to the detection and localization of FGF receptors 1 and 2 (FGFR1-2), the specific membrane proteins that bind to FGFs. ${ }^{15,16}$ The present study stresses the importance of the FGF signaling pathways for organ regeneration in lizards, and amniotes in general.

\section{Materials and methods}

In the present study we have utilized fixed tissues originally prepared in previous studies from adults of the lizard Podarcis muralis. ${ }^{17}$ The experiments were conducted following the Italian guidelines for animal care and handling (art. 5, DL 116/92). The lizards were kept in a terrarium at $25-33^{\circ} \mathrm{C}$ with a photoperiod of $12-14$ hours of light, and they were fed with a diet of mealworms, fruit-flies and fly larvae. The lizards were maintained at $4-6^{\circ} \mathrm{C}$ for 30 minutes to produce hypothermia and numbness, and hindlimb amputation was done in the distal part of the tight close to the knee, using a sharp razor blade, after anesthetizing the lizards with ethyl ether. Stump samples with their healing blastema from 15-16days $(n=3)$, and 25-26days $(n=3)$ post-amputation, were selected among others for the present study, and were fixed in $4 \%$ paraformaldehyde in $0.1 \mathrm{M}$ Phosphate buffer at $\mathrm{pH} 7.4$ at $0-4^{\circ} \mathrm{C}$ for 5hours, rinsed in buffer for about 30minutes, dehydrated in ethanol $(70 \%, 80 \%, 95 \%)$, infiltrated in xylene, and embedded in wax. The tissues were sectioned at $6-8-8 \mathrm{~m}$ in the longitudinal plane using a microtome, and were collected and dried on glass slides. Sample sections were stained with HematoxylinEosin (H \& E) for the general histological study.

The immunological detection of FGF1,2 and of FGFR1,2 was done using an antibody against FGF1 produced in rabbit stimulated 
after injection of FGF1 obtained from Sigma. ${ }^{18}$ The rabbit FGF2 antibody was purchased from Sigma (F3393). The antibody against FGFR1 was a rabbit polyclonal antibody directed against an epitope of the C-terminus of the receptor. ${ }^{19}$ The FGFR2 antibody from rabbit was obtained from Santa Cruz (c-17, the beck isoform), and is directed against an epitope located in the $\mathrm{C}$-terminal cytoplasmic side of the receptor. All the antibodies were generously provided from Dr. Frank Lovicu, University of Sydney, Australia, and the rabbit FGFR1 antibody 803 derived from Dr. A Baird laboratory Wittier Institute, La Jolla, CA, USA, ${ }^{19}$

Sections were de-waxed with xylene, rehydrated and pre-incubated for 30 minutes in Buffer containing 5\% of Normal Goat Serum and 2\% Bovine Serum Albumin (BSA). For the detection of FGFs and their receptors, the sections were incubated for 4-5 hours at room temperature with the rabbit antibodies at a dilution 1:100 in $0.1 \mathrm{M}$ Phosphate buffer containing $2 \%$ BSA, while in control sections the primary antibody was omitted. After rinsing for 10minutes with 3 changes in the Buffer, the sections were incubated for $1 \mathrm{~h}$ at room temperature with a Tetramethyl Rhodamine Isotiocyanate (TRITC, Sigma, USA) labeled anti-Rb secondary antibodies (dilution 1:150). After the final rinsing, the sections were mounted in Fluoromount (Sigma, USA), and later observed under a fluorescent microscope. For western blot analysis, three regenerating tail blastemas of $2-3 \mathrm{~mm}$ in length, and three normal tails were collected and immediately homogenized as indicated below. For the separation of the epidermis from the dermis and inner tissues, other five normal and five regenerating tails of $2-3 \mathrm{~mm}$ in length were utilized for protein extraction. On this purpose, small pieces of regenerating tail were incubated in $5 \mathrm{~mm}$ EDTA in phosphate buffered saline (PBS) for $3-5 \mathrm{~min}$ at $50^{\circ} \mathrm{C}$ and $2-4 \mathrm{~min}$ in cold PBS. The epidermis was separated from the dermis by dissection using a stereomicroscope and homogenized. Tissues were homogenized in $8 \mathrm{M}$ urea $/ 50 \mathrm{mM}$ Tris- $\mathrm{HCl}$ at $\mathrm{pH} 7.6$ containing $0.1 \mathrm{M}$ 2-mercaptoethanol $/ 1 \mathrm{mM}$ dithiothreithol/ and $1 \%$ protease inhibitor (SIGMA, St Louis, USA). The particulate matter was removed by centrifugation at $10,000 \mathrm{~g}$ for $5 \mathrm{~min}$, and protein concentration was assayed by the Bradford method before electrophoresis.

For the electrophoretic migration, $40 \mathrm{~g}$ of proteins were loaded in each lane and separated in 15\% SDS-polyacrylamide gels (SDSPAGE) using the MiniProtean III electrophoresis apparatus (Bio$\mathrm{Rad})$. For western blotting, the proteins separated in SDS-PAGE were initially transferred to nitrocellulose paper, and they were stained with Ponceau red to verify the protein transfer. The protein bands were incubated with primary anti-FGF1,2 or FGFR1,2 rabbit antibodies while in controls, the primary antibodies were omitted. Detection was performed using the enhanced chemiluminescence procedure developed by Amersham (ECL, Plex Western Blotting System, GE Healthcare, UK).

\section{Results}

In the selected limb blastemas presented in this study, the regenerating limb at 16 days after amputation appeared as a small outgrowth covered by a shiny dark epidermis inset in (Figure 1). Histologically it showed a thick wound (regenerating) epidermis with a thin corneous layer that covered underneath a loose connective tissue here termed a regenerating blastema for analogy with the blastema of the regenerating tail details in. ${ }^{6,7,17}$ However, differently from the tail blastema, the limb blastema is generally destined to form a scarring outgrowth in the following days, and it grows to about $1 \mathrm{~mm}$ before turning into a scaled outgrowth. Among the proximal tissues included in the limb stump at 16 days after the amputation, some fragmented and repairing muscles were present, and the sectioned femur showed a compact lamellar bone in the diaphysis while its proximal, untouched epiphysis showed in the cavity of the secondary ossification center the presence of some cartilaginous cells (Figure 1). At 26 days the analyzed blastema was still covered by a thick wound epidermis with a thicker corneous layer and contained a dense and irregular connective tissue, most composed by elongated fibroblasts in the superficial, apical region, and an even denser connective tissues formed by spindle-shaped fibroblasts in proximal regions, in contact or penetrating among the original tissues of the stump (Figure 1C-G). Numerous blood vessels were sparsely distributed among fibroblasts, and mitotic figures of dividing fibroblasts were rare to absent.

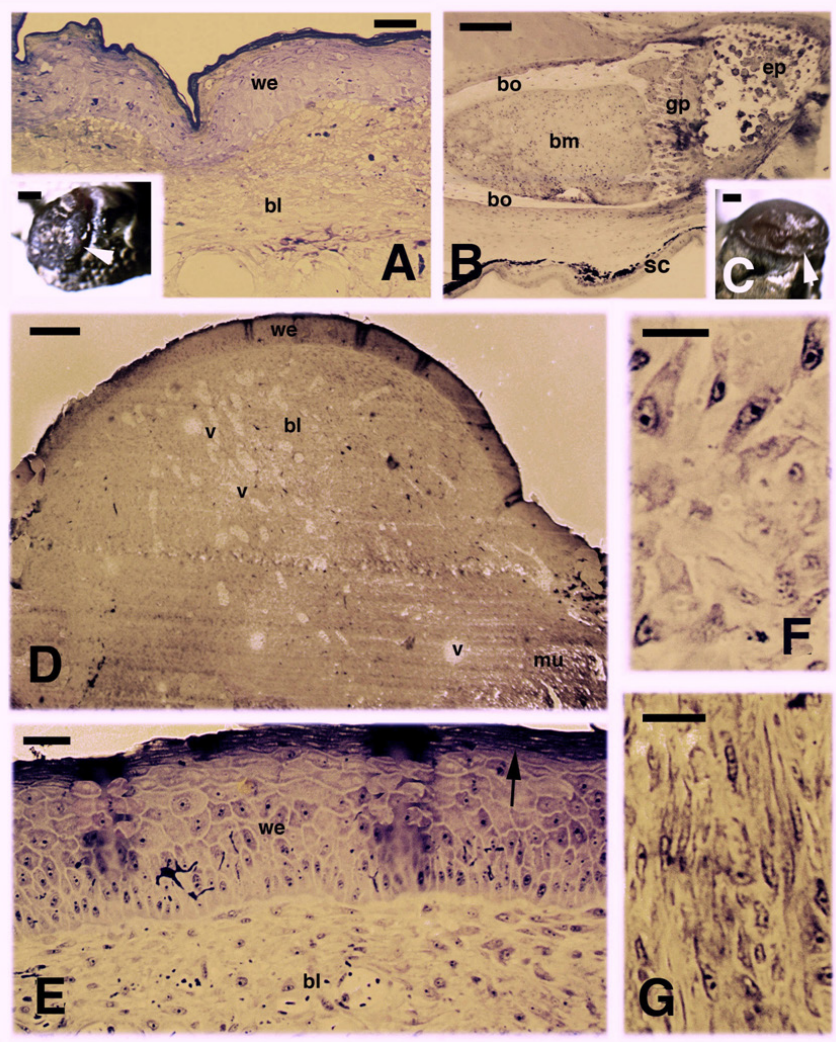

Figure I Histological examples of regenerating limb at 16 days (A, B) and 26 days (C-G) post-amputation. $\mathbf{A}$, at 16 days the amputated limb appears partially covered by a scab (arrowheads in the inset, Bar $0.5 \mathrm{~mm}$ ). Histological section shows a thick wound epidermis covering a blastema made of a loose connective tissue. Bar, $10 \mathrm{~m}$. B, detail on the femur remnant contacting the blastema, and showing the degenerated cartilage in the epiphysis. Bar, $50 \mathrm{~m}$. C, limb stump covered by a roundish blastema (arrowhead). Bar, $0.5 \mathrm{~mm}$. D, histology of the blastema showing a loose connective tissue with sparse blood vessels. Bar, $50 \mathrm{~m}$. E, detail on the thick wound epidermis with a multilayered corneous layer (arrow). Bar, I0m. F, a detail of the superficial blastema shows numerous fibroblasts. Bar, $10 \mathrm{~m}$. G, a more proximal region of the blastema, near the stump tissues, shows elongated fibroblasts forming a denser connective tissue. Bar, I0m. Legends: bl, blastema; bm, bone marrow; bo, femur compact bone (diaphysis); ep, epiphysis; gp, growth plate (metaphysis); mu, stump muscles; sc, scale; v, blood vessels; we, wound (regenerating) epidermis.

Western blotting observations from the analyzed healing limbs of 25-26 days post-amputation showed main immunopositive bands at 48-50, 67-70 and 75-77kDa for FGF1 and FGF2, the latter showing 
more intensely labeled bands at $60-65$ and $75-80 \mathrm{kDa}$ (Figure 2, first and second lanes). No or very weak bands were seen at 19$22 \mathrm{kDa}$ (the closer MW to the expected MW of monomer and active forms for FGF1-2). Controls were negative (Figure 2, third lane). After separation of the regenerating epidermis from the underlying connective tissues indicated for simplification as "regenerated dermis" in (Figure 2), western blot immunodetection for receptors showed in the regenerating (wound) epidermis two bands at 55 and $58-60 \mathrm{kDa}$ for FGFR1 (Figure 2, fourth lane) and a band around 120kDa and $18 \mathrm{kDa}$ in the regenerating dermis for FGFR1 (Figure 2, fifth lane). The regenerated epidermis showed a main band around $80 \mathrm{kDa}$, and weaker at 85 and 57-60kDa for FGFR2 (Figure 2 sixth lane), while almost no bands or a very weak band at $47 \mathrm{kDa}$ for FGFR2 was seen in the regenerating dermis (Figure 2, seventh lane)
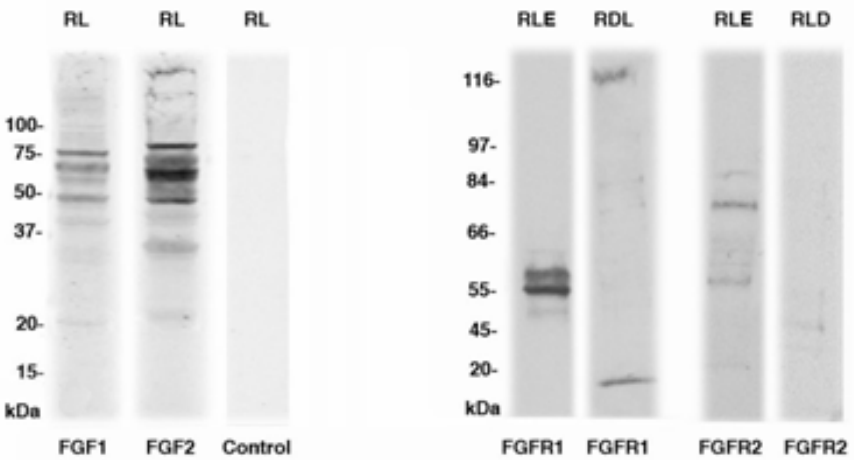

Figure 2 Western blotting for FGFI, FGF2, FGFRI and FGFR2 in the regenerating limb (RL), in the regenerated limb epidermis (RLE) and regenerated limb dermis (+inner tissues, indicated as RLD). The MW is indicated in the ordinate in kilo Daltons $(\mathrm{kDa})$.

Immunolabeling for FGF1 and FGF2 showed a more intense immunofluorescence in the wound epidermis and less in the blastema with both FGF1 and FGF2 at 16days post-amputation, although FGF1 showed a higher labeling also in blastema cells (Figure 3A) (Figure 3B). Periosteal cells covering the lamellar bone of the femur and chondroblasts of its repairing distal end, originally transected, also showed some immunolabeling for FGF1 and FGF2 at 16days (Figure 3C). Immunofluorescence for both FGFs lowered in the healing tissues of the limb blastema at 26days post-amputation, especially in the wound epidermis covering the outgrowth (Figure 3D) (Figure $3 \mathrm{E})$. An un-even immunofluorescence remained in the repairing or regenerating muscles, in the periosteum and in chondrocytes of the healing femur at its distal, previously transected ends (data not shown). No labeling was seen in control sections, aside a non-specific auto-fluorescence due to the erythrocytes present in blood vessels and in some auto fluorescence in the corneous layer of the epidermis (Figure 3F).

The immunolocalization at 16 days post-amputation for FGFR1 showed an immune labeled wound epidermis while sparse and weakly labeled cells were observed in cells located in the connective tissue located underneath the wound epidermis, here, indicated as blastema (Figure 4A) (Figure 4B). The FGFR1 labeling was instead present among the repairing muscles and in the cartilage cells and periosteum of the distal, healing region of the initially transected bone of the femur (Figure 4C) (Figure 4D). Interestingly, sparse immune labeled cells were also detected in the cartilaginous cells present in the proximal epiphysis of the femur, a region untouched by the amputation (Figures 1B) (Figure 4E). Control sections appeared immunonegative
(Figure 4F). Differently from 16 days, almost no immune labeling for FGFR1 was seen in the blastema at 26days post-amputation, in both the wound epidermis and the underlying blastema (Figure 5A). However in the cartilage of the regenerating femur and in that of its proximal epiphyses, the latter untouched by the amputation, sparse cartilaginous labeled cells for FGFR1 were still seen even at 26days post-amputation (Figure 5B) (Figure 5C). A similar observation was also made for FGFR2, that appeared present in the apical wound epidermis at 16days but was low to absent in the blastema (Figure 5D). Sparse labeled cells were however detected in the proximal epiphyses of the femur (not hit by the transaction) at 16 and 26days post-injection also for FGFR2 (Figure 5E). Immunofluorescence for FGFR2 lowered or disappeared at 26days in both wound epidermis and blastema cells, despite the non-specific auto fluorescence of blood vessels (Figure 5F).

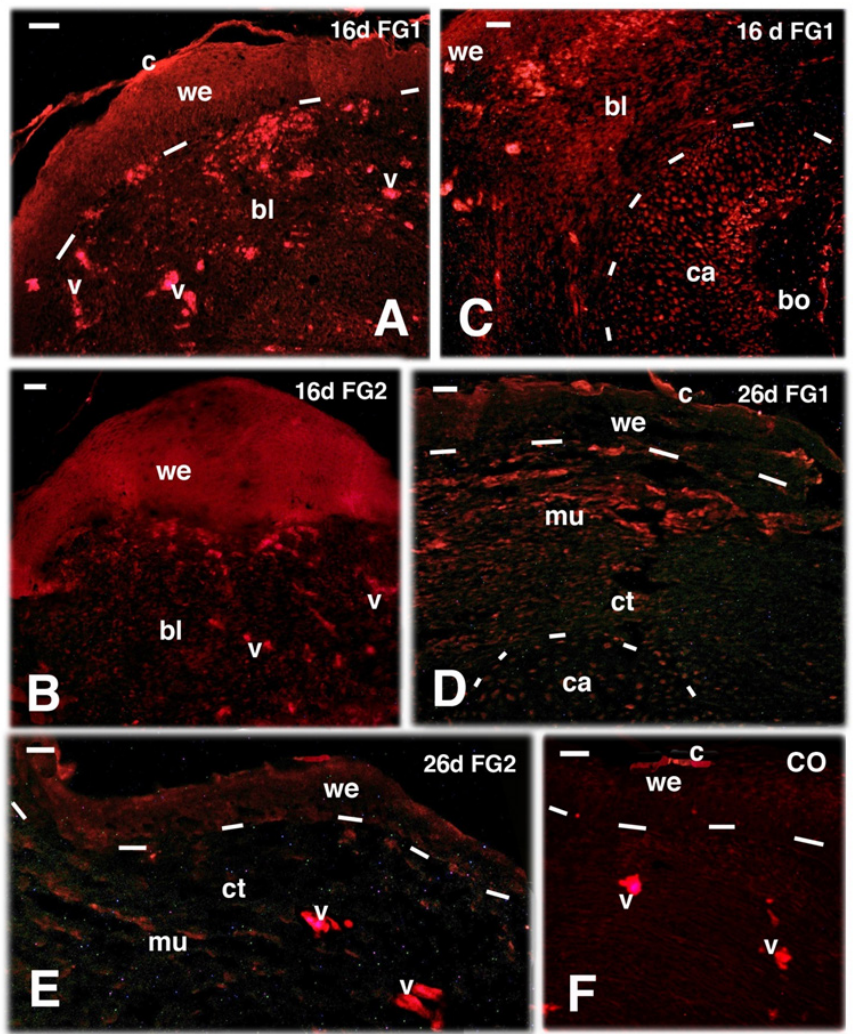

Figure 3 TRITC immunofluorescence for FGFI (A, C, D) and FGF2 (B, E) and control $(\mathbf{F})$ in regenerating limbs at 16 and 26 days (d) post-amputation. Bars indicate $10 \mu \mathrm{m}$ in all figures. A, at 16 days both the wound epidermis and sparse cells in the blastema are immunofluorescent ( $\mathrm{v}$ indicate nonspecific fluorescence in blood vessels). B,thick and immunofluorescent wound epidermis at 16 days. C, sparse immunofluorescence in both the wound epidermis and underlying blastema connective and the end of the transacted femur (outlined by dashes) showing labelled cells (chondroblasts and periosteal cells). D, lowering of immunofluorescence in the repairing connective and muscles of the blastema, that almost disappears in the wound epidermis aside non-specific labeling of fragments of the corneous layer). E, lowering to disappearing of immunofluorescence in the wound epidermis and underlying connective blastema. F, immunonegative control (CO). Legends: bl, blastema; bo, bone tissue (femur); c, corneous layer; ca, cartilage tissue/Periosteal cells on the transacted femur; $\mathrm{mu}$, repairing/regenerating muscles; $\mathrm{v}$, blood vessels (filled with auto-fluorescent erythrocytes); we, wound epidermis. The dashes underline the wound epidermis in $\mathbf{A}, \mathbf{D}, \mathbf{E}, \mathbf{F}$, and they outline the cartilaginous apex of the transected femur in $\mathbf{C}$ and $\mathbf{D}$. 

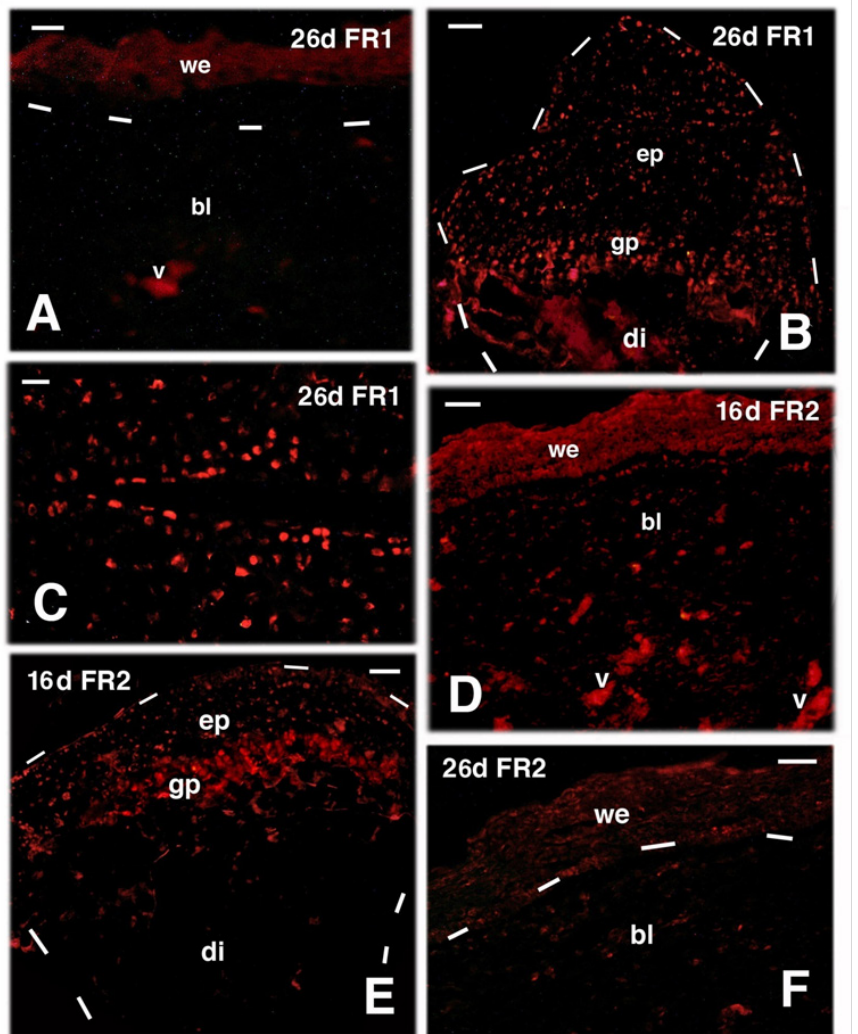

Figure 4 TRITC immunofluorescence for FGFRI (FRI) at I6 days (d) postamputation.A,the wound epidermis and sparse cells in the blastema are labelled (fragments of the corneous layer are non-specifically fluorescent) 16 days posttrauma. Bar, $20 \mathrm{~m}$. B, apical blastema showing the immunofluorescent wound epidermis and diffuse labelled connective and repairing muscle cells of the underlying blastema. Bar, $20 \mathrm{~m}$. C, higher magnification view of repairing tissues within the blastema at 16 days post-lesion, showing immunofluorescence in both muscles and interspersed connective tissue cells. Bar, 10m. D, at 16 days post-trauma, underneath the wound epidermis the connective forming the blastema and repairing muscles, contains sparse immunolabeled cells. Labelled cartilaginous and periosteal cells (outlined by dashes) are in continuity with the femur bone that is not labelled. Bar, $20 \mathrm{~m}$. E, detail of labeled cells located in the proximal cartilaginous epiphysis (untouched by the transection) of the femur (outlined by dashes). Asterisks indicate a non-specific fluorescence of the trabecular bone. Bar, $10 \mathrm{~m}$. F, immuno-negative control section of apical blastema (dashes underline the wound epidermis). Bar, $10 \mathrm{~m}$. Legends: bl, blastema; bo, bone of the femur; ca, repairing/reactive cartilage; bo, bone; gp, growth plate; mu, regenerated muscles; v, autofluorescent blood vessels; we, wound epidermis. The dashes outline the shape of the cartilage outgrowth in $\mathrm{D}$ and of the epiphyses in $\mathrm{E}$.

\section{Discussion}

The regeneration of limb outgrowths of different length in lizards depends from some conditions, including trimming the exposed femur after the shrinkage of the stump connective and muscle tissues during the first days post-trauma, and keeping the temperature above $25^{\circ} \mathrm{C} .{ }^{3-5}$ In our selected cases, we analyzed the best conditions when the stump was fully re-epithelial zed at 16 days after the injury and formed a blastema-like outgrowth at 26 days that was accumulating fibroblasts and formed ascarring connective tissues rich in irregular extracellular fibrils.

In the cases here analyzed, FGF1-2 and FGFR1-2 appear present where cell proliferation is still active at 15-16 days post-injury, especially in the regenerating epidermis, in sparse blastema cells, and mainly in the repairing muscle cells or in the periosteum and cartilaginous epiphyses of the femur. ${ }^{18,20}$ FGF immune labeling tends to disappear later on when the proliferation in the limb decreases and only few tissues such a muscle and cartilageare still repairing. ${ }^{6,7,17,20}$ Cell proliferation and FGF immune labeling is also abolished in scarring tails after cauterization, an intervention that drives the tail to form a scar instead of regenerates into a new tail. ${ }^{6-8,10}$ The importance of FGFs and FGFRs for lizard regeneration confirms previous observations, ${ }^{10}$ and the numerous data available for anamniotes such as amphibians and fish, two groups of vertebrates where tissue and organ regeneration is even higher than in lizards..$^{21-26}$ The researches on amphibians and fish have indeed shown that FGFs and their receptors are mitogenic growth factors for the regeneration of the amphibian limb or the fish fin, mimicking most of the activity of the Neurotrophic Factor. ${ }^{22,27}$ FGFs are released from nerves and the spinal cord, and maintain cell proliferation in the blastema, especially FGF1 while FGF2 is present and release from the Apical Epidermal Cap and maintains cells proliferation in concert with other signaling proteins. $^{22-24,27,28}$

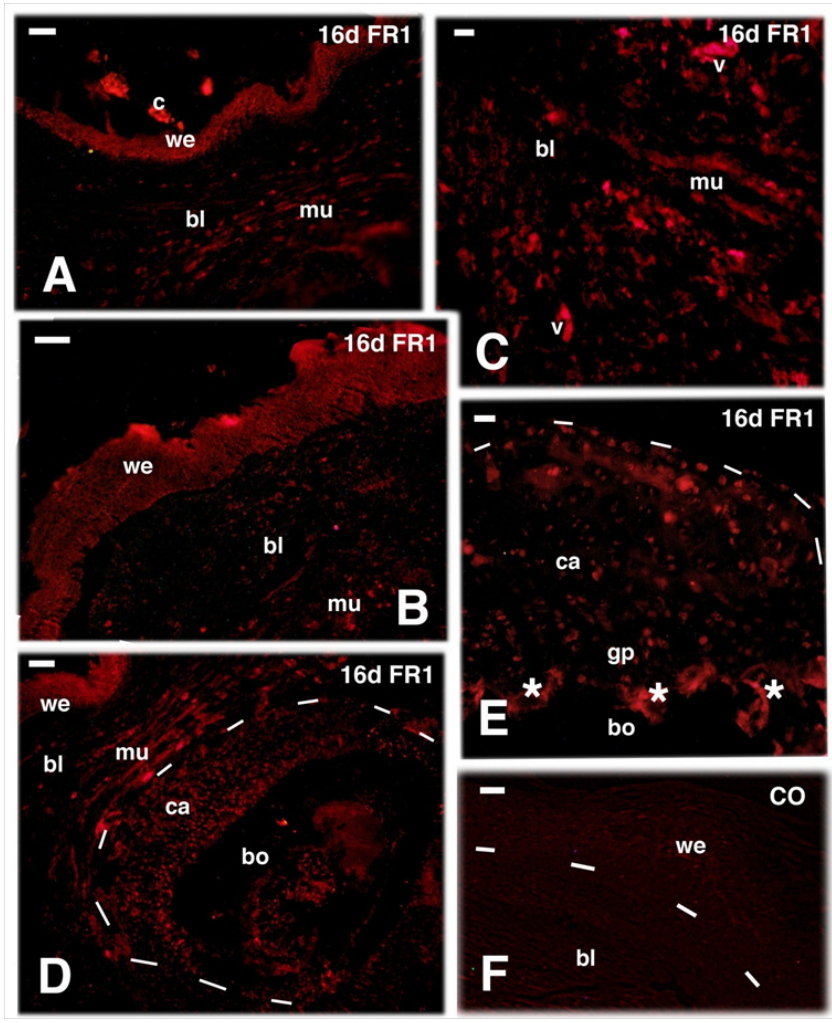

Figure 5 TRITC immunofluorescence for FGFRI (FRI) at 26 days (d) and FGFR2 (FR2) at 16 and 26 days (d) post-amputation. A, most of the wound epidermis and blastema are immunonegative for FGFRI at 26 days posttrauma. Bar, $10 \mathrm{~m}$. B, sparse immunolabeled cells of the proximal cartilaginous epiphysis and growth plate of the femur 26 days after amputation (untouched by amputation). Bar, $10 \mathrm{~m}$. C, detail of labelled cartilaginous cells of the uninjured femur epiphysis. Bar, $20 \mathrm{~m}$. D, immunofluorescent labeled wound epidermis and few blastema connectives at 16 days. Bar, $20 \mathrm{~m}$. E, cartilaginous epiphysis of the untouched femur 16 days post-amputation. Bar, $20 \mathrm{~m}$. F, low labeling at 26 days, almost disappeared in the wound epidermis and few labeled cells in the underlying connective blastema. Bar, 20m. Legends: bl, blastema; $\mathrm{di}$, diaphysis (femur); ep, epiphysis (femur); gp, growth plate (metaphysis). $v$, auto fluorescent blood vessels; we, wound epidermis. Dashes underline the epidermis in $\mathbf{A}$ and $\mathbf{F}$, the shape of the femur in $\mathbf{B}$ and $\mathbf{E}$. 
The initial presence of FGF and their receptors in the first 2 weeks of limb healing in lizards likely stimulates the wound epidermis to proliferate in order to cover the exposed tissues of the stump. FGF2 also stimulated angiogenesis and the rate of blastema growth in the tail, ${ }^{11,21}$ a process that is also active in the initial phases of limb regeneration. ${ }^{6,7}$ However, the intense and persisting inflammation in the connective tissues, bones and muscles of the limb due to a massive accumulation of immune cells, macrophages and lymphocytes, in the stump and initial blastema, leads to scarring. The detected immunopositive bands for FGFs and their receptors at 50-80kDa, a range outside that expected for FGF (16 to $27 \mathrm{kDa}$, see ${ }^{15,16,19}$ ), indicate polymerization of lower bands or degradation of the High MW precursors in our material. The lack of the active protein bands at 18$22 \mathrm{kDa}$ for FGF1 and FGF2 and of higher MW bands for their receptors in the limb, further confirms the decrease or complete disappearing of these signaling proteins during these later stages of limb recovery. FGFs are instead present in the regenerating tail where FGF appears stimulating tissue regeneration. ${ }^{10}$ Another indication of the important role of FGFs for tail regeneration in lizards derives from experiments where the administration of FGF and/or FGFRs inhibitors before and during blastema formation determines failure of tail regeneration. ${ }^{11-13}$ In conclusion FGF and FGFR1-2 are part of the essential signaling pathways that sustain the growth of the blastema and regeneration of cartilage, muscle, and the new epidermis in lizards and vertebrates in general.

\section{Acknowledgements}

None.

\section{Conflict of interest}

The author declares no conflict of interest.

\section{References}

1. Marcucci E. La rigenerazione degli arti nei rettili. Boll Soc Natur. $1925 ; 38: 8-17$

2. Marcucci E. Il potere rigenerativo degli arti nei Rettili. Ricerche sperimentale sopra alcune specie di Sauri. Arch Zool Ital. 1930;14:237252 .

3. Barber LW. Correlations between wound healing and regeneration in fore-limbs and tails of lizards. Anat Rec. 1944;89(4):441-453.

4. Bellairs AA, Bryant SV. Effects of amputation of limbs and digits of lacertid lizards. Anat Rec. 1968;161(4):489-495.

5. Bellairs AA, Bryant SV. Autotomy and regeneration in reptiles. In: Billet GCF, Maderson PFA editors. Biology of the Reptilia. (Vol 15B), NY, USA: John Wiley, Sons; 1985. p. 302-410.

6. Alibardi L. Morphological and cellular aspects of tail and limb regeneration in lizard: a model system with implications for tissue regeneration in mammals. Adv Anat Embr Cell Biol. 2010;207:1-109.

7. Alibardi L. Ultrastructural features of the process of wound healing after tail and limb amputation in lizard. Acta Zoolica. 2010;91(3):306-318.

8. Alibardi L. Ultrastructural observations on the scarring process in the cauterized tail and the amputated limb of lizard. Trends Dev Biol. $2013 ; 7: 15-24$

9. Vitulo N, Dalla Valle L, et al. Transcriptome analysis of the regenerating tail versus the scarring limb in lizard reveals pathways leading to successful versus unsuccessful organ regeneration in amniotes. Dev Dyn. 2017;246(2):116-134.
10. Alibardi L. Observations on FGFs immunoreactivity in the regenerating tail blastema and in the limb and tail scars of lizard suggests that FGFs are required for regeneration. Belg J Zool. 2012;142:23-38.

11. Yadav M, Anusree P, Desai I, et al. Influence of extraneous FGF-2 and its antagonist aniti-FGF-2 on the progress of tail regeneration in Hemidactylus flaviviridis. Ind J Fund Appl Sci. 2012;2:164-172.

12. Pillai A, Desai I, Balakrishnan S. Pharmacological inhibition of FGFR1 signaling attenuates the progression of tail regeneration in the northern house gecko Hemidactylus flaviviridis. Int J LifeSc Bt Pharm Res. 2013;2(4):263-278.

13. Naraynan A. The initiation and progression of tail regeneration in northern house gecko Hemidactylus flaviviridis at role of Fibroblast Growth Factor 2 (Fgf2). Biochips Tissue Chips. 2015;1:1-7.

14. Hutchins ED, Markov GJ, Eckalbar WL, et al. Transcriptomic analysis of tail regeneration in the lizard Anolis carolinensis reveals activation of conserved vertebrate developmental and repair mechanisms. Plos One. 2014;9(8):e105004.

15. Beenken A, Mohammadi M. The FGF family: biology, pathophysiology, and therapy. Nat Rev Drug Discov. 2009;8(3):235-253.

16. Ornitz DM, Itoh N. The fibroblast growth factor signaling pathway. Wiley Interdiscip Rev Dev Biol. 2015;4(3):215-266.

17. Alibardi L. Immunolocalization indicates that both original and regenerated lizard tail tissues contain populations of long retaining cells, putative stem/progenitor cells. Micr Res Techn. 2015;78(11):1032-1045.

18. Schultz MW, Chamberlain CG, de Jongh RU, et al. Acidic and basic FGF in ocular media and lens: implications for lens polarity and growth patterns. Development. 1993;118(1):117-126.

19. Hanneken A, Maher PA, Baird A. High affinity immunoreactive FGF receptors in the extracellular matrix of vascular endothelial cellsimplications for the modulation of FGF-2. J. Cell Biol. 1995;128(6):12211228.

20. Alibardi L. Cell proliferation in the amputated limb of lizard leading to scarring is reduced compared to the regenerating tail. Acta Zool. 2016;98(2):170-180.

21. Zenjari C, Boilly-Marer BY, Desbiens X, et al. Experimental evidence for FGF1 control of blastema cell proliferation during limb regeneration of the amphibian Pleurodeles waltl. Int J Dev Biol. 1996;40(5):965-971.

22. Mescher AL. The cellular basis of limb regeneration in urodeles. Int $J$ Dev Biol. 1996;40(4):785-795.

23. Ferretti P, Zhang F, Santos-Ruiz L, et al. FGF signaling and blastema growth during amphibian tail regeneration. Int J Dev Biol. 2001;45 (SI):S127-S128.

24. Lee Y, Grill S, Sanchez A, et al. Fgf signaling instructs positiondependent growth rate during zebrafish fin regeneration. Development. 2005;132(23):5173-5183.

25. McKusker C, Bryant SV, Gardiner DM. The axolotl limb blastema: cellular and molecular mechanism driving blastema formation and limb regeneration in tetrapods. Regeneration (Oxf). 2015;2(2):54-71.

26. Tanaka EM. The molecular and celluklar choreography of appendage regeneration. Cell. 2016;165(7):1598-1608.

27. Singer M. On the nature of the neurotrophic phenomenon in urodele limb regeneration. Am Zool. 1978;18(4):829-841.

28. Poulin ML, Patrie KM, Botelho MJ, et al. Heterogeinity in the expression of fibroblast growth factor receptors during limb regeneration in newts (Notophthalmus viridescens). Development. 1993;119(2):353-361. 